\title{
Some applications of minimax and topological degree to the study of the Dirichlet problem for elliptic partial differential equations
}

\author{
by Leszek GȩBa and Tadeusz Pruszko (Gdańsk)
}

Abstract. This paper treats nonlinear elliptic boundary value problems of the form (1)

$$
L[u]=p(x, u) \quad \text { in } \Omega \subset \mathbb{R}^{n}, \quad u=D u=\ldots=D^{m-1} u \quad \text { on } \partial \Omega,
$$

in the Sobolev space $W_{0}^{m, 2}(\Omega)$, where $L$ is any selfadjoint strongly elliptic linear differential operator of order $2 m$. Using both topological degree arguments and minimax methods we obtain existence and multiplicity results for the above problem.

1. Introduction. In this paper we shall be concerned with the existence of nontrivial solutions of problem (1). To this end we associate with (1) a completely continuous vector field $\Phi: W_{0}^{m, 2}(\Omega) \rightarrow W_{0}^{m, 2}(\Omega)$ or a functional $I: W_{0}^{m, 2}(\Omega) \rightarrow \mathbb{R}$ of class $C^{1}$ in such a way that the set of all generalized solutions of $(1)$ is the set of all zeros of $\Phi$ or the set of all critical points of $I$. The connection between $I$ and $\Phi$ is such that the gradient of the nonlinear part of $I$ is the $L$-compact part of $\Phi$. Using Ambrosetti and Rabinowitz's Mountain Pass Theorem [3] we prove two theorems on the existence of nontrivial critical points of $I$ and as a consequence we obtain an existence result for generalized nontrivial solutions of (1). On the other hand, using both the critical points of $I$ and some topological degree arguments, we obtain the existence of multiple nontrivial solutions.

Extensive applications of critical points to problem (1) have been considered in the case when $L$ is the Laplacian or an operator of order 2 (see, for instance, [3], [4], [13]; for a full list of references cf. [12]). Our theorems for an operator $L$ of order $2 m$ give some generalizations of the previous ones, or are obtained under different assumptions on $p(\cdot, \cdot)$.

\section{Preliminaries}

I. An elliptic differential operator. Let $\Omega$ be a bounded domain in $\mathbb{R}^{n}$ 
with smooth boundary $\partial \Omega$ and let

$$
L[u]=\sum_{|\alpha|,|\beta| \leq m}(-1)^{|\beta|} D^{\beta}\left(a_{\alpha \beta}(x) D^{\alpha} u(x)\right)
$$

be a differential operator defined for every $C^{2 m}$-function $u: \Omega \rightarrow \mathbb{R}$. We shall need the following conditions:

$L[\cdot]$ is strongly elliptic in $\Omega$, i.e. there exists a $C_{0}>0$ such that for every $x \in \mathbb{R}^{n}, \xi \in \mathbb{R}^{n}$

$$
\sum_{|\alpha|,|\beta|=m} a_{\alpha \beta}(x) \xi^{\alpha} \xi^{\beta} \geq C_{0}|\xi|^{2 m},
$$

$a_{\alpha \beta}: \Omega \rightarrow \mathbb{R}$ are bounded $C^{m}$-functions, $a_{\alpha \beta}=a_{\beta \alpha}$ for every $|\alpha|,|\beta| \leq m$ and $a_{\alpha \beta}$ are uniformly continuous for $|\alpha|,|\beta|=m$.

We denote by $C_{0}^{\infty}(\Omega)$ the space of all smooth functions in $\Omega$ which have compact support in $\Omega$. Integrating by parts yields that for every $u, \varphi \in$ $C_{0}^{\infty}(\Omega)$

$$
\int_{\Omega} L[u](x) \varphi(x) d x=\int_{\Omega} \sum_{|\alpha|,|\beta| \leq m} a_{\alpha \beta}(x) D^{\alpha} u(x) D^{\beta} \varphi(x) d x .
$$

Thus with the operator $L[\cdot]$ we can associate the bilinear form

$$
(u, \varphi) \rightarrow \int_{\Omega} L[u(x)] \varphi(x) d x \quad \text { for } u, \varphi \in C_{0}^{\infty}(\Omega) .
$$

That form is continuous in $C_{0}^{\infty}(\Omega)$ with the norm

$$
\|u\|_{m}=\left(\sum_{|\alpha| \leq m} \int\left(D^{\alpha} u\right)^{2} d x\right)^{1 / 2} .
$$

We denote by $W_{0}^{m, 2}(\Omega)$ the closure of $C_{0}^{\infty}(\Omega)$ in the norm $\|\cdot\|_{m}$. It is well known that $W_{0}^{m, 2}(\Omega)$ is a Hilbert space and we call it the Sobolev space; we denote the scalar product in $W_{0}^{m, 2}(\Omega)$ by $\langle\cdot, \cdot\rangle_{m}$. By the definition of $W_{0}^{m, 2}(\Omega)$,

(2.4) There is a unique continuous, bilinear form $B: W_{0}^{m, 2}(\Omega) \times$ $W_{0}^{m, 2}(\Omega) \rightarrow \mathbb{R}$ such that $B(u, \varphi)=\int_{\Omega} L[u] \varphi d x$ for every $u, \varphi \in$ $C_{0}^{\infty}(\Omega)$.

We call $B(\cdot, \cdot)$ the Dirichlet form of $L$.

A bilinear form $B$ on $W_{0}^{m, 2}(\Omega)$ is called coercive if there is $C_{1}>0$ such that $B(u, u) \geq C_{1}\|u\|_{m}^{2}$ for every $u \in W_{0}^{m, 2}(\Omega)$.

In our paper we will use the following three main theorems:

(2.5) (Sobolev Embedding Lemma [5]). Let $\Omega$ be a bounded domain in $\mathbb{R}^{n}$ with smooth boundary. If $u \in W_{0}^{m, 2}(\Omega)$ then $u \in L^{t}(\Omega)$ where $t \in$ 
$[1,2 n /(n-2 m)](n>2 m)$ and there is a constant $C_{2}>0$ such that

$$
\|u\|_{L^{t}(\Omega)} \leq C_{2}\|u\|_{m}
$$

for all $u \in W_{0}^{m, 2}(\Omega)$. Moreover, the embedding $j: W_{0}^{m, 2}(\Omega) \hookrightarrow L^{t}(\Omega)$ is completely continuous.

The same assertion holds for $n \leq 2 m$ and $t \in[1, \infty)$.

(2.6) (Gårding Inequality [6]). If the assumptions (2.2), (2.3) hold, then there exist constants $C_{3}, K_{0}>0$ such that $B(u, u) \geq C_{3}\|u\|_{m}^{2}-K_{0}\|u\|_{0}^{2}$ for all $u \in W_{0}^{m, 2}(\Omega)$.

(2.7) (Lax-Milgram Theorem [5]). If $B$ is a continuous, bilinear and coercive form in the Hilbert space $W_{0}^{m, 2}(\Omega)$ then there exists a unique isomorphism $A: W_{0}^{m, 2}(\Omega) \rightarrow W_{0}^{m, 2}(\Omega)$ such that $\langle A(u), \varphi\rangle_{m}=B(u, \varphi)$ for every $u, \varphi \in W_{0}^{m, 2}(\Omega)$.

II. Leray-Schauder degree. Let $E$ be a real Banach space. We call a continuous map $F: E \rightarrow E$ completely continuous if for every bounded subset $X \subset E$ the image $F(X)$ is relatively compact. A map $f: E \rightarrow$ $E$ is a completely continuous vector field if $f(x)=x-F(x)$, where $F$ is completely continuous. For such $f$ and for every open, bounded $U \subset E$ with $0 \notin f^{-1}(\partial U)$, the Leray-Schauder degree $\operatorname{deg}(f, U, 0) \in \mathbb{Z}$ exists.

We will use some properties of the degree (for more details see [1], [9]):

(2.8) (Existence). If $\operatorname{deg}(f, U, 0) \neq 0$, then there exists $x \in U$ with $f(x)=0$.

(2.9) (Additivity). If $U_{1}, U_{2}$ are open subsets of $E$ and $U_{1} \subset U, U_{2} \subset U$, $U_{1} \cap U_{2}=\emptyset, f(x) \neq 0$ for $x \in U \backslash\left(U_{1} \cup U_{2}\right)$ then $\operatorname{deg}(f, U, 0)=\operatorname{deg}\left(f, U_{1}, 0\right)+$ $\operatorname{deg}\left(f, U_{2}, 0\right)$.

(2.10) (Homotopy). If $f_{1}, f_{2}: E \rightarrow E$ are vector fields joined by a homotopy $h(t, x)=x-H(t, x)$, where $H:[0,1] \times E \rightarrow E$ is completely continuous and $h(t, x) \neq 0$ for $(t, x) \in[0,1] \times \partial U$, then $\operatorname{deg}\left(f_{1}, U, 0\right)=\operatorname{deg}\left(f_{2}, U, 0\right)$.

From (2.9) and (2.10) we have:

(2.11) If $f: E \rightarrow E$ is a completely continuous vector field which is differentiable at its isolated zero $x_{0}$ and $f^{\prime}\left(x_{0}\right): E \rightarrow E$ is invertible then there exists $r>0$ such that

$$
\operatorname{deg}\left(f, K\left(x_{0}, r\right), 0\right)=\operatorname{deg}\left(f^{\prime}\left(x_{0}\right), K(0, r), 0\right),
$$

where $K\left(x_{0}, r\right) \subset E$ is the open ball with centre at $x_{0}$ and radius $r$.

We will also use the following Leray-Schauder formula:

(2.12) If $T: E \rightarrow E$ is linear, completely continuous and such that the vector field $I-T: E \rightarrow E$ is invertible then

$$
\operatorname{deg}(I-T, U, 0)=(-1)^{m}
$$


where $m=\sum_{\mu>1} m(\mu)$ and $m(\mu)$ is the multiplicity of the eigenvalue $\mu$ of $T$.

III. Mountain Pass Theorem. Let $I: E \rightarrow \mathbb{R}$ be a $C^{1}$-functional, where $E$ is a real Banach space. We say $I$ satisfies the Palais-Smale condition if

(PS) any sequence $\left(u_{m}\right) \subset E$ for which $I\left(u_{m}\right)$ is bounded and $I^{\prime}\left(u_{m}\right) \rightarrow 0$ (in $E^{*}$ ) as $m \rightarrow \infty$ has a convergent subsequence.

We say $v \in E$ is a critical point of the functional $I: E \rightarrow \mathbb{R}$ if $I^{\prime}(v)=0$.

(2.14) (Mountain Pass Theorem [3]). Suppose $I \in C^{1}(E, \mathbb{R})$ satisfies (PS). Let $I(0)=0$ and suppose that

$\left(\mathrm{I}_{1}\right)$ there are constants $\varrho, \gamma>0$ such that $\left.I\right|_{\partial K(0, \varrho)} \geq \gamma$,

$\left(\mathrm{I}_{2}\right)$ there is $e \in E \backslash K(0, \varrho)$ such that $I(e) \leq 0$.

Then $I$ has a critical value $c \geq \gamma$ and there exists $u_{0} \neq 0$ which is a critical point of $I$.

3. Existence and multiplicity results. We shall need the following assumptions on a function $p: \bar{\Omega} \times \mathbb{R} \rightarrow \mathbb{R}:$

(3.1) $\quad p$ is a Carathéodory function, i.e. $p(\cdot, \xi)$ is continuous for every $\xi \in \mathbb{R}$ and $p(x, \cdot)$ is measurable for every $x \in \bar{\Omega}$;

$|p(x, \xi)| \leq a_{1}+a_{2}|\xi|^{s}$ with $a_{1}, a_{2}>0$ and $0 \leq s<(n+2 m) /(n-2 m) ;$

(3.4) there exist constants $\mu>2$ and $r>0$ such that if $|\xi|>r$ then $0 \leq \mu P(x, \xi) \leq \xi p(x, \xi)$ where $P(x, t)=\int_{0}^{t} p(x, \xi) d \xi$

(3.5) $\quad 0 \leq \mu P(x, \xi) \leq \xi p(x, \xi)$ for $0<r \leq|\xi| \leq 2 K r$ where $\mu>2, K>1$ and $K^{\mu-2} \int_{\Omega} P(x, v(x)) d x>B(v, v)$ for some $v \in W_{0}^{m, 2}(\Omega)$ such that $r \leq v(x) \leq 2 r, x \in \bar{\Omega}$;

(3.6) $\quad p_{\xi}(x, \cdot) \in C(\mathbb{R}, \mathbb{R}),\left|p_{\xi}(x, \xi)\right| \leq a_{3}+a_{4}|\xi|^{\varrho}$ where $a_{3}, a_{4}>0, \varrho \leq s-1$ and $p_{\xi}(x, \cdot)$ is the differential of $p(x, \cdot)$.

In what follows, for a bilinear form $B: W_{0}^{m, 2}(\Omega) \times W_{0}^{m, 2}(\Omega) \rightarrow \mathbb{R}$ and for a Carathéodory function $p: \bar{\Omega} \times \mathbb{R} \rightarrow \mathbb{R}$ we will consider the functional $I: W_{0}^{m, 2}(\Omega) \rightarrow \mathbb{R}$ given by

$$
I(u)=\frac{1}{2} B(u, u)-\int_{\Omega} P(x, u(x)) d x .
$$

THEOREM 1. If $B: W_{0}^{m, 2}(\Omega) \times W_{0}^{m, 2}(\Omega) \rightarrow \mathbb{R}$ is a bilinear, continuous, coercive form and the function $p: \bar{\Omega} \times \mathbb{R} \rightarrow \mathbb{R}$ satisfies (3.1)-(3.4), then the 
functional $I: W_{0}^{m, 2}(\Omega) \rightarrow \mathbb{R}$ given by (3.7) is of class $C^{1}$ and it has at least one nonzero critical point.

The following version of Theorem 1 for a bounded function $p: \bar{\Omega} \times \mathbb{R} \rightarrow \mathbb{R}$ is also useful for boundary value problems.

Theorem 2. If $B: W_{0}^{m, 2}(\Omega) \times W_{0}^{m, 2}(\Omega) \rightarrow \mathbb{R}$ is a bilinear, continuous, coercive form and the bounded function $p: \bar{\Omega} \times \mathbb{R} \rightarrow \mathbb{R}$ satisfies assumptions (3.1)-(3.3), (3.5) then the functional $I: W_{0}^{m, 2}(\Omega) \rightarrow \mathbb{R}$ given by (3.7) is $C^{1}$ and it has at least one nonzero critical point.

We next consider the nonlinear Dirichlet problem

$$
\begin{cases}(L+a)[u]=p(x, u(x)), & x \in \Omega, \\ \left.D^{\alpha} u\right|_{\partial \Omega}=0, & |\alpha| \leq m-1,\end{cases}
$$

where $L[\cdot]$ is a differential operator satisfying assumptions (2.1)-(2.3) and $a \in \mathbb{R}$.

A function $u \in W_{0}^{m, 2}(\Omega)$ is a generalized solution of the Dirichlet problem (3.8) if

$$
B(u, \varphi)=\int_{\Omega} p(x, u(x)) \varphi(x) d x \quad \text { for } \varphi \in W_{0}^{m, 2}(\Omega),
$$

where $B(\cdot, \cdot)$ is the Dirichlet form of the operator $L+a$ (see (2.4)).

THEOREM 3. If the function $p: \bar{\Omega} \times \mathbb{R} \rightarrow \mathbb{R}$ satisfies (3.1)-(3.4) (or (3.1)(3.3), (3.5)) then there exists a constant $K>0$ such that for every $a>K$ problem (3.8) has a nonzero generalized solution.

If the function $p: \bar{\Omega} \times \mathbb{R} \rightarrow \mathbb{R}$ is differentiable in its second variable, then with every nonzero generalized solution $v \in W_{0}^{m, 2}(\Omega)$ of problem (3.8) we will associate the linear Dirichlet problem

$$
\begin{cases}(L+a)[u]=p_{\xi}(x, v(x)) u, & x \in \Omega \\ \left.D^{\alpha} u\right|_{\partial \Omega}=0, & |\alpha| \leq m-1 .\end{cases}
$$

Theorem 4. Let $p: \bar{\Omega} \times \mathbb{R} \rightarrow \mathbb{R}$ satisfy (3.1)-(3.6) and suppose that

(*) for every nonzero generalized solution $v \in W_{0}^{m, 2}(\Omega)$ of problem (3.8) the function $u=0$ is a unique generalized solution of $(3.9)_{v}$.

Then problem (3.8) has at least two different, nonzero generalized solutions provided $a>K_{0}$.

4. Nemytskiı operator. We will use the well known

(4.1) Lemma (see [12], and also [14] for characterization of the continuity of the Nemytskiil operator). Let $\Omega \subset \mathbb{R}^{n}$ be bounded and open, and let $g$ : $\bar{\Omega} \times \mathbb{R} \rightarrow \mathbb{R}$ be a continuous function such that $|g(x, \xi)| \leq a_{1}+a_{2}|\xi|^{r / q}$ for 
$(x, \xi) \in \bar{\Omega} \times \mathbb{R}, r, q \geq 1, a_{1}, a_{2}>0$. Then the map $G: L^{r}(\Omega) \rightarrow L^{q}(\Omega)$ such that

$$
[G(u)](x)=g(x, u(x))
$$

is well defined and continuous.

We call $G$ the Nemytski乞 operator associated with the function $g$.

(4.2) Lemma. If conditions (3.1), (3.2) hold for a function $g: \bar{\Omega} \times \mathbb{R} \rightarrow \mathbb{R}$ then the operator $G: W_{0}^{m, 2}(\Omega) \rightarrow L^{q}(\Omega)$ with $q=2 n /(n+2 m)$ given by (4.1.1) for every $u \in W_{0}^{m, 2}(\Omega)$ is well defined and completely continuous.

Pro of. Consider $G$ as a composition

$$
W_{0}^{m, 2}(\Omega) \stackrel{j}{\longrightarrow} L^{t}(\Omega) \stackrel{G_{1}}{\longrightarrow} L^{t / s}(\Omega) \stackrel{i}{\hookrightarrow} L^{q}(\Omega),
$$

where $G_{1}$ is the Nemytskil operator with the same formula as $G, j$ is the completely continuous embedding from the Sobolev Lemma (2.5) with $t=$ $2 n /(n-2 m)$ and $i$ is the inclusion. From (3.2) and Lemma (4.1) we deduce that $G_{1}$ is continuous, which means $G$ is completely continuous.

(4.3) LemmA. Let $\Omega, g$ and $G$ be as in Lemma (4.1). Let $r>q \geq 1$ and suppose that

(4.3.1) the derivative $g_{\xi}(x, \cdot) \in C(\mathbb{R}, \mathbb{R})$ exists and there are $a_{3}, a_{4}>0$ such that

$$
\left|g_{\xi}(x, \xi)\right| \leq a_{3}+a_{4}|\xi|^{\mu}, \quad \text { where } \mu \leq(r-q) / q .
$$

Then the Nemytskiu operator $G$ is differentiable and

$$
[D G(u)] \varphi(x)=g_{\xi}(x, u(x)) \varphi(x) .
$$

Pro of. We have to show that

$$
\|G(u+\varphi)-G(u)-[D G(u)] \varphi\|_{L^{q}}<\varepsilon\|\varphi\|_{L^{r}} \quad \text { if }\|\varphi\|_{L^{r}}<\delta .
$$

Using the Mean Value Theorem and the Hölder inequality we have

$$
\begin{aligned}
\frac{1}{\|\varphi\|_{L^{r}}}\left[\int_{\Omega}\left|g(x, u(x)+\varphi(x))-g(x, u(x))-g_{\xi}(x, u(x)) \varphi(x)\right|^{q} d x\right]^{1 / q} \\
\leq \frac{1}{\|\varphi\|_{L^{r}}}\left[\int_{\Omega}\left|g_{\xi}(x, u(x)+\vartheta(x) \varphi(x))-g_{\xi}(x, u(x))\right|^{q}|\varphi(x)|^{q} d x\right]^{1 / q} \\
\leq \frac{\left[\left[\int_{\Omega}\left(|\varphi(x)|^{q}\right)^{r / q} d x\right]^{q / r}\right]^{1 / q}}{\left[\int_{\Omega}|\varphi(x)|^{r} d x\right]^{1 / r}} \\
\times\left[\left\|\left|g_{\xi}(x, u(x)+\vartheta(x) \varphi(x))-g_{\xi}(x, u(x))\right|^{q}\right\|_{L^{r /(r-q)}}\right]^{1 / q} .
\end{aligned}
$$

All we have to show now is

$$
\|\varphi\|_{L^{r}}<\delta \Rightarrow\left\|\left|g_{\xi}(x, u(x)+\vartheta(x) \varphi(x))-g_{\xi}(x, u(x))\right|^{q}\right\|_{L^{r /(r-q)}}<\varepsilon .
$$


We will use an obvious criterion:

$$
\begin{aligned}
& \left\|\varphi_{n}\right\|_{L^{t}(\Omega)} \rightarrow 0 \text { as } n \rightarrow \infty \text { if and only if for every subsequence } \\
& \left\{\varphi_{n_{k}}\right\} \text { there exists }\left\{\varphi_{n_{k_{l}}}\right\} \subset\left\{\varphi_{n_{k}}\right\} \text { such that: } \\
& 1^{\mathrm{O}} \varphi_{n_{k_{l}}} \rightarrow 0 \text { a.e., } \\
& 2^{\mathrm{o}} \text { there exists } w \in L^{t}(\Omega) \text { such that }\left|\varphi_{n_{k_{l}}}(x)\right| \leq w(x) .
\end{aligned}
$$

Let $\left\|\varphi_{n}\right\|_{L^{r}(\Omega)} \rightarrow 0$ and let $\varphi_{n_{k}}$ be any subsequence of $\varphi_{n}$. Since $g_{\xi}(x, \cdot) \in C(\mathbb{R}, \mathbb{R})$ and $\varphi_{n_{k}} \rightarrow 0$ a.e. we have

$$
g_{\xi}\left(x, u(x)+\vartheta(x) \varphi_{n_{k_{l}}}(x)\right)-g_{\xi}(x, u(x)) \rightarrow 0 \quad \text { a.e. }
$$

Moreover,

$$
\begin{aligned}
\mid g_{\xi}\left(x, u(x)+\vartheta(x) \varphi_{n_{k_{l}}}\right. & (x))-g_{\xi}(x, u(x)) \mid \\
& \leq\left|g_{\xi}\left(x, u(x)+\vartheta(x) \varphi_{n_{k_{l}}}(x)\right)\right|+\left|g_{\xi}(x, u(x))\right| \\
& \leq 2 a_{3}+a_{4}|u(x)|^{\mu}+a_{4}\left|u(x)+\varphi_{n_{k_{l}}}(x)\right|^{\mu} \\
& \leq 2 a_{3}+a_{5}\left|u(x)+\varphi_{n_{k_{l}}}(x)\right|^{\mu}=w(x) \in L^{r q /(r-q)}
\end{aligned}
$$

because $\mu \leq(r-q) / q$. Using (4.3.2) we have

$$
\left\|\varphi_{n}\right\|_{L^{r}} \rightarrow 0 \Rightarrow\left\|\left|g_{\xi}\left(x, u(x)+\vartheta(x) \varphi_{n}(x)\right)-g_{\xi}(x, u(x))\right|^{q}\right\|_{L^{r /(r-q)}} \rightarrow 0 \text {. }
$$

This finishes proof of the lemma.

5. Reformulation of the Dirichlet problem. Recall that $u \in$ $W_{0}^{m, 2}(\Omega)$ is a (generalized) solution of problem (3.8) if $u$ is a solution of the following equation, which we call the generalized Dirichlet problem for (3.8):

$$
B(u, \varphi)=\int_{\Omega} p(x, u(x)) \varphi(x) d x \quad \text { for } \varphi \in W_{0}^{m, 2}(\Omega),
$$

where $B: W_{0}^{m, 2}(\Omega) \times W_{0}^{m, 2}(\Omega) \rightarrow \mathbb{R}$ is the Dirichlet form associated with the operator $L+a$.

In the rest of our paper, whenever we use minimax methods we will associate with the generalized Dirichlet problem (5.1) a special functional $I: W_{0}^{m, 2}(\Omega) \rightarrow \mathbb{R}$, which is of class $C^{1}$. If we use topological degree methods we will work with a completely continuous vector field $f: W_{0}^{m, 2}(\Omega) \rightarrow$ $W_{0}^{m, 2}(\Omega)$.

We start with a proposition which is a consequence of the Riesz Theorem:

(5.2) Proposition. Let $E$ be a Hilbert space with a scalar product $\langle\cdot, \cdot\rangle$ and let $\Phi: E \rightarrow \mathbb{R}$ be a functional of class $C^{1}$. Then there exists exactly one continuous map $\nabla \Phi: E \rightarrow E$ such that

$$
\left[\Phi^{\prime}(u)\right](v)=\langle\nabla \Phi(u), v\rangle \quad \text { for every } u, v \in E .
$$

We call the map $\nabla \Phi: E \rightarrow E$ the gradient of $\Phi$. 
(5.3) Lemma. Let $p: \bar{\Omega} \times \mathbb{R} \rightarrow \mathbb{R}$ satisfy (3.1), (3.2), let $G: W_{0}^{m, 2}(\Omega) \rightarrow$ $L^{q}(\Omega)$ with $q=2 n /(n+2 m)$ be the operator given in Lemma (4.2) and let $j_{q}: W_{0}^{m, 2}(\Omega) \rightarrow L^{q}(\Omega)$ be the Sobolev embedding. Then the functional $J: W_{0}^{m, 2}(\Omega) \rightarrow \mathbb{R}$ defined by

$$
J(u)=\int_{\Omega}^{u(x)} \int_{0}^{u} p(x, t) d t d x
$$

is $C^{1}$ and its gradient $\nabla J: W_{0}^{m, 2}(\Omega) \rightarrow W_{0}^{m, 2}(\Omega)$ is completely continuous with

$$
\nabla J=j_{q}^{*} \circ G
$$

where $j_{q}^{*}: L^{q}(\Omega) \rightarrow W_{0}^{m, 2}(\Omega)$ is the adjoint operator to $j_{q}\left(j_{q}^{*}(u)=w \Leftrightarrow\right.$ $\left.\langle w, \varphi\rangle_{m}=\left\langle u, j_{q}(\varphi)\right\rangle_{0}\right)$.

Proof. We show first $J \in C^{1}\left(W_{0}^{m, 2}(\Omega), \mathbb{R}\right)$. Consider $J$ as a composition:

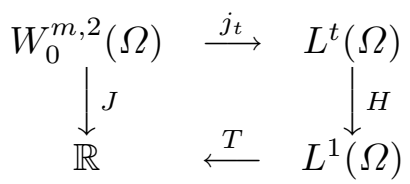

where $j_{t}: W_{0}^{m, 2}(\Omega) \rightarrow L^{t}(\Omega)$ is the Sobolev embedding with $t=2 n /(n-$ $2 m), T$ is the integration operator, $T(w)=\int_{\Omega} w(x) d x$, and $H$ is the Nemytskiu operator associated with $P(x, u(x))=\int_{0}^{u(x)} p(x, t) d t$. $H$ is well defined because by $(3.2),|P(x, \xi)| \leq a+a|\xi|^{s+1}$ and $s+1 \leq 2 n /(n-2 m)$.

By Lemma (4.3) with $g_{\xi}=p, g=P, r=t, q=1$ we find that $J$ is differentiable and $J^{\prime}(u) \varphi=\int_{\Omega} p(x, u(x)) \varphi(x) d x$ for every $\varphi \in W_{0}^{m, 2}(\Omega)$. Moreover, we have the commutative diagram

$$
\begin{array}{llll}
W_{0}^{m, 2}(\Omega) & & \\
W_{0}^{m, 2}(\Omega) & \searrow & \longleftarrow & \\
j_{q}^{*} & L^{q}(\Omega)
\end{array}
$$

where $G$ is the operator given in Lemma (4.2) $(q=2 n /(n+2 m))$. By Lemma (4.1), $G$ is continuous and $J^{\prime}$ is continuous. By Lemma (4.2), $G$ is completely continuous, and hence so is $\nabla J$.

Let $B: W_{0}^{m, 2}(\Omega) \times W_{0}^{m, 2}(\Omega) \rightarrow \mathbb{R}$ be the Dirichlet form appearing in (5.1) and let $J: W_{0}^{m, 2}(\Omega) \rightarrow \mathbb{R}$ be the $C^{1}$-functional from Lemma (5.3). With the generalized Dirichlet problem (5.1) we associate the $C^{1}$-functional $I: W_{0}^{m, 2}(\Omega) \rightarrow \mathbb{R}$ defined by

$$
I(u)=\frac{1}{2} B(u, u)-J(u) \quad \text { for } u \in W_{0}^{m, 2}(\Omega) .
$$


If the form $B(\cdot, \cdot)$ is coercive then (by the Lax-Milgram theorem) there exists a unique continuous linear isomorphism $A: W_{0}^{m, 2}(\Omega) \rightarrow W_{0}^{m, 2}(\Omega)$ such that

$$
B(u, \varphi)=\langle A(u), \varphi\rangle_{m} \quad \text { for } u, \varphi \in W_{0}^{m, 2}(\Omega) .
$$

Then with the generalized Dirichlet problem (5.1) we can associate the following completely continuous vector field $f: W_{0}^{m, 2}(\Omega) \rightarrow W_{0}^{m, 2}(\Omega)$ :

$$
f=\mathrm{id}-A^{-1} \circ(\nabla J) \text {. }
$$

(5.7) Lemma. Let $p: \bar{\Omega} \times \mathbb{R} \rightarrow \mathbb{R}$ satisfy (3.1), (3.2). Then: $u \in W_{0}^{m, 2}(\Omega)$ is a solution of $(5.1) \Leftrightarrow u$ is a critical point of $I: W_{0}^{m, 2}(\Omega) \rightarrow \mathbb{R}$

(5.7.2) if $p$ satisfies $(3.6)$ and $B(\cdot, \cdot)$ is coercive then $u \in W_{0}^{m, 2}(\Omega)$ is a solution of $(3.8) \Leftrightarrow u$ is a zero of the completely continuous vector field $f=\mathrm{id}-A^{-1} \circ(\nabla J)$.

Proof. (5.7.1) If $u \in W_{0}^{m, 2}(\Omega)$ and $I^{\prime}(u)=0$ then $B(u, \varphi)=$ $\int_{\Omega} p(x, u(x)) \varphi(x) d x$, that is, $u$ is a solution of (3.8).

(5.7.2) If $f(u)=u-A^{-1} \circ \nabla J(u)=0$ then $A(u)=\nabla J(u),\langle A(u), \varphi\rangle_{m}=$ $\langle\nabla J(u), \varphi\rangle_{m}$ for $\varphi \in W_{0}^{m, 2}(\Omega)$, and

$$
B(u, \varphi)=\left\langle j_{2}^{*} \circ G, \varphi\right\rangle_{m}, \quad B(u, \varphi)=\int_{\Omega} p(x, u(x)) \varphi(x) d x,
$$

where $G$ is the Nemytskiu operator associated with $p$.

\section{Proofs of theorems.}

I. Proof of Theorem 1. We will use the Mountain Pass Theorem (cf. (2.14)). We first list the steps of the proof.

(6.1) Assumptions (3.1), (3.2) imply that $I \in C^{1}\left(W_{0}^{m, 2}(\Omega), \mathbb{R}\right)$.

(6.2) Assumptions (3.1)-(3.3) imply that $I(0)=0$ and

$\left(\mathrm{I}_{1}\right)$ there are constants $\varrho, \gamma>0$ such that $I(u) \geq \gamma$ for $u \in \partial K(0, \varrho)$.

(6.3) Assumptions (3.1), (3.2) and (3.4) imply that $\left(\mathrm{I}_{2}\right)$ there exists $u \in W_{0}^{m, 2}(\Omega) \backslash K(0, \varrho)$ such that $I(u) \leq 0$.

(6.4) Assumptions (3.1), (3.2) imply that every bounded sequence $\left\{u_{k}\right\} \subset$ $W_{0}^{m, 2}(\Omega)$ such that $I^{\prime}\left(u_{k}\right) \rightarrow 0$ has a convergent subsequence.

(6.5) The functional $I$ satisfies condition (PS).

We claim that $\left(\frac{1}{2} B(u, u)\right)^{\prime} \varphi=B(u, \varphi)$. Indeed,

$$
\left|\frac{1}{2} B(u+\varphi, u+\varphi)-\frac{1}{2} B(u, u)-B(u, \varphi)\right|
$$




$$
\begin{aligned}
& =\left|\frac{1}{2} B(u, u)+\frac{1}{2} B(u, \varphi)+\frac{1}{2} B(\varphi, u)+\frac{1}{2} B(\varphi, \varphi)-\frac{1}{2} B(u, u)-B(u, \varphi)\right| \\
& =\left|\frac{1}{2} B(\varphi, \varphi)\right| \leq \frac{1}{2} C_{1}\|\varphi\|_{m} \leq \varepsilon\|\varphi\|_{m} \quad \text { if }\|\varphi\|_{m} \leq 2 \varepsilon / C_{1} .
\end{aligned}
$$

Since $B$ is continuous, $\frac{1}{2} B(\cdot, \cdot) \in C^{1}\left(W_{0}^{m, 2}(\Omega), \mathbb{R}\right)$. By Lemma (5.3), $J \in$ $C^{1}\left(W_{0}^{m, 2}(\Omega), \mathbb{R}\right)$, which means $I \in C^{1}\left(W_{0}^{m, 2}(\Omega), \mathbb{R}\right)$.

We now check the other assumptions of the Mountain Pass Theorem.

$I(0)=0$ is obvious.

$\left(\mathrm{I}_{1}\right)$ By $(3.2),(3.3)$ for $\delta>0$

$$
\exists C_{4}>0 \forall x \in \Omega \forall|\xi|>\delta \quad|P(x, \xi)| \leq C_{4}|\xi|^{s+1} .
$$

By $(3.3)$

$$
\forall \varepsilon>0 \exists \delta>0 \forall x \in \Omega \forall|\xi| \leq \delta \quad|P(x, \xi)| \leq C_{5} \varepsilon|\xi|^{2} .
$$

Now,

$$
\begin{aligned}
& \int_{\Omega}|P(x, u(x))| d x \leq C_{5} \varepsilon \int_{\Omega}|u(x)|^{2} d x+C_{4} \int_{\Omega}|u(x)|^{1+s} d x \\
& \quad \leq C_{5} \varepsilon C_{3}^{2}\|u\|_{m}^{2}+C_{4} C_{3}\|u\|_{m}^{s+1} \leq\|u\|_{m}^{2}\left(C_{5} C_{3} \varepsilon+C_{4}\|u\|_{m}^{s-1}\right) C_{3} \leq \varepsilon\|u\|_{m}^{2}
\end{aligned}
$$

provided $\|u\|_{m} \leq\left(C_{5} \varepsilon\right)^{1 /(s-1)} / C_{4}$. That means $J(u)=o\left(\|u\|_{m}^{2}\right)$. By (3.4), $I(u)=\frac{1}{2} B(u, u)-J(u) \geq C_{1}\|u\|_{m}^{2}-o\left(\|u\|_{m}^{2}\right)$. Hence there exist $\varrho, \alpha>0$ such that $I(u) \geq \alpha$ for every $u \in \partial K(0, \varrho)$.

$\left(\mathrm{I}_{2}\right)$ We first show $P(x, \xi) \geq b_{1}|\xi|^{\mu}-b_{2}$ for $|\xi|>r>0$, where $b_{1}, b_{2}>0$. By $(3.4), \mu / t \leq p(x, t) / P(x, t)$ for $t>r>0$, and

Therefore

$$
\int_{r}^{\xi} \frac{\mu}{t} d t \leq \int_{r}^{\xi} \frac{p(x, t)}{P(x, t)} d t .
$$

$$
\mu(\ln \xi-\ln r) \leq \ln P(x, \xi)-\ln P(x, r) .
$$

Hence there exist $b_{1}, b_{2}>0$ such that $b_{1}|\xi|^{\mu} \leq P(x, \xi)$ for $|\xi|>r$ and $b_{1}|\xi|^{\mu}-b_{2} \leq P(x, \xi)$ for every $\xi$. The proof is the same when we start with $t<0$. Now,

$$
I(t u)=\frac{1}{2} B(t u, t u)-J(t u) \leq \frac{t^{2}}{2} C_{1}\|u\|_{m}^{2}-b_{1} t^{\mu} \int_{\Omega}|u(x)|^{\mu} d x+b_{2}|\Omega| .
$$

Since $\mu>2$ we get $I(t u) \rightarrow-\infty$ as $t \rightarrow \infty$. Thus there exists $u \in W_{0}^{m, 2}(\Omega)$, $u \notin K(0, \varrho)$, such that $I(u) \leq 0$.

Before we show that $I$ satisfies (PS) we must prove:

(6.6) LEMMA. If $\left\{u_{k}\right\}$ is bounded in $W_{0}^{m, 2}(\Omega)$ and $I^{\prime}\left(u_{k}\right) \rightarrow 0$ then $\left\{u_{k}\right\}$ has a convergent subsequence.

Proof. Since $I^{\prime}(u) \varphi=\langle A(u), \varphi\rangle_{m}-J^{\prime}(u) \varphi$ for $\varphi \in W_{0}^{m, 2}(\Omega)$, we have $A^{-1} I^{\prime}(u)=u-A^{-1} J^{\prime}(u)$ and $u_{k}=A^{-1} I^{\prime}\left(u_{k}\right)+A^{-1} J^{\prime}\left(u_{k}\right)$. Since $J^{\prime}$ is 
completely continuous, and $\left\{u_{k}\right\}$ is bounded, it follows that $J^{\prime}\left(u_{k}\right)$ has a convergent subsequence, and hence so does $\left\{u_{k}\right\}$.

By Lemma (6.6) it is sufficient to show that if $\left|I\left(u_{k}\right)\right|<M$ for each $k \in \mathbb{N}$ and $I^{\prime}\left(u_{k}\right) \rightarrow 0$ then $\left\{u_{k}\right\}$ is bounded. Let

$$
T=-P\left(x, u_{k}(x)\right)+\frac{1}{\mu} p\left(x, u_{k}(x)\right) u_{k}(x) .
$$

Then

$$
\begin{aligned}
M+\frac{\left\|u_{k}\right\|_{m}}{\mu} \geq & I\left(u_{k}\right)-\frac{I^{\prime}\left(u_{k}\right) u_{k}}{\mu} \geq \frac{1}{2} B\left(u_{k}, u_{k}\right)-\frac{1}{\mu} B\left(u_{k}, u_{k}\right)+\int_{\Omega} T d x \\
& \geq\left(\frac{1}{2}-\frac{1}{\mu}\right) C_{2}\left\|u_{k}\right\|_{m}^{2}+\int_{\left\{x \in \Omega \mid u_{k}(x)>r\right\}} T d x+\int_{\left\{x \in \Omega \mid u_{k}(x) \leq r\right\}} T d x .
\end{aligned}
$$

The second term on the right hand side is positive by (3.4), and the third is bounded. Therefore $\left\{u_{k}\right\}$ is bounded.

We have just shown that all assumptions hold, i.e. by the Mountain Pass Theorem the functional $I$ has a nonzero critical point.

II. Proof of Theorem 2. As before Theorem 2 is a consequence of the Mountain Pass Theorem. Assumptions (3.1)-(3.3), (3.5) imply that $I$ defined by (3.7) satisfies conditions (6.1), (6.2) and (6.4), hence it is sufficient to show:

(a) condition $\left(\mathrm{I}_{2}\right)$, and

(b) $\left|I\left(u_{k}\right)\right| \leq M$ for each $k \in \mathbb{N}$ and $I^{\prime}\left(u_{k}\right) \rightarrow 0$ imply that $\left\{u_{k}\right\}$ is bounded.

To prove (a) observe that, as in the proof of (6.3),

$$
\begin{gathered}
\mu(\ln \xi-\ln r) \leq \ln P(x, \xi)-\ln P(x, r), \\
(\xi / r)^{\mu} P(x, r) \leq P(x, \xi) \quad \text { for } x \in \Omega, r \leq \xi \leq 2 K r .
\end{gathered}
$$

Let $v \in W_{0}^{m, 2}(\Omega)$ be a function as in assumption (3.5). Then $r \leq K v(x) \leq$ $2 K r$ and so by (3.5)

$$
\begin{aligned}
I(K v) & \leq \frac{1}{2} K^{2} B(v, v)-K^{\mu} \int_{\Omega} \frac{v(x)^{\mu}}{r} P(x, r) d x \\
& \leq K^{2}\left[\frac{1}{2} B(v, v)-K^{\mu-2} \int_{\Omega} P(x, r) d x\right] \leq 0 .
\end{aligned}
$$

Therefore condition $\left(\mathrm{I}_{2}\right)$ is satisfied for $u=K v$.

To prove (b), as before in (6.5), we start with the inequality

$$
M+\left\|u_{k}\right\|_{m} / \mu \geq I\left(u_{k}\right)-\left[I^{\prime}\left(u_{k}\right)\right]\left(u_{k}\right) / \mu
$$


(for $k$ sufficiently large) or in the equivalent form

$$
\begin{aligned}
M+\frac{\left\|u_{k}\right\|_{m}}{\mu}+ & \int_{\Omega}^{u} \int_{0}^{u(x)} p(x, t) d t d x \\
& \geq\left(\frac{1}{2}-\frac{1}{\mu}\right) B\left(u_{k}, u_{k}\right)+\frac{1}{\mu} \int_{\Omega} p\left(x, u_{k}(x)\right) u_{k}(x) d x .
\end{aligned}
$$

Since $p$ is bounded: $|p(x, \xi)| \leq N$ for $x \in \bar{\Omega}, \xi \in \mathbb{R}$, we have

$$
M+\left\|u_{k}\right\|_{m}\left(N / \mu+2 N C_{I}\right) \geq(1 / 2-\mu) C_{2}\left\|u_{k}\right\|_{m}^{2},
$$

where $N>1$ and $\|u\|_{L^{1}} \leq C_{I}\|u\|_{m}$. Therefore $\left\{u_{k}\right\}$ is bounded. Now we see that all assumptions of the Mountain Pass Theorem are satisfied and the proof is complete.

III. Pr o of of Theorem 3. By the Gårding inequality (2.6) if $a>K_{0}$ then $B$ is coercive. By Theorem 1 the functional $I$ has a nonzero critical point $u \in W_{0}^{m, 2}(\Omega)$, which (by Lemma (5.7)) is a generalized solution of problem (3.8).

IV. Proof of Theorem 4. Since by assumption the function $p$ : $\bar{\Omega} \times \mathbb{R} \rightarrow \mathbb{R}$ is bounded, so is the map $\nabla J=j_{2}^{*} \circ G$ (cf. (5.3.1)). Now in virtue of (5.7.2) the set $\Gamma(I)$ of all solutions of (3.8) is compact. On the other hand, the vector field $\Phi(u)=u-\left[A^{-1} \circ \nabla J\right](u)$ is differentiable and for every $w \in \Gamma(I)$

$$
\left[\Phi^{\prime}(w)\right](v)=v-\left[A^{-1} \circ j_{2}^{*} \circ G^{\prime}(w)\right](v) .
$$

By Lemma (4.3), $\left[G^{\prime}(w) v\right](x)=p_{\xi}(x, w(x)) v(x)$, so in virtue of assumptions $(*),(3.3)$, and Lemma $(4.3), \Phi^{\prime}(w)$ is invertible and therefore every zero of $\Phi$ is isolated. Hence the compact set $\Gamma(I)$ is finite. Put $\Gamma(I) \backslash\{0\}=$ $\left\{u_{1}, \ldots, u_{s}\right\}$. By Theorem 2 this is a non-empty set. Next we make use of the Leray-Schauder degree:

$1^{\circ}$ Choose $R>0$ such that $\operatorname{Im}\left(A^{-1} \circ \nabla J\right) \subset K(0, R)$. Then we have the linear homotopy

$$
H(t, u)=u-t\left(A^{-1} \circ \nabla J\right)(u) \quad \text { for } t \in[0,1], u \in W_{0}^{m, 2}(\Omega)
$$

and by $(2.10)$

$$
\operatorname{deg}(\Phi, K(0, R), 0)=\operatorname{deg}(\mathrm{id}, K(0, R), 0)=1 .
$$

$2^{\circ}$ If $u_{i} \in \Gamma(I) \backslash\{0\}$ then by (2.11), (2.12) there exists $r_{i}>0$ such that $\operatorname{deg}\left(\Phi, K\left(u_{i}, r_{i}\right), 0\right)=\operatorname{deg}\left(\Phi^{\prime}\left(u_{i}\right), K\left(0, r_{i}\right), 0\right)= \pm 1$.

$3^{\circ}$ Since $\left[\Phi^{\prime}(0)\right](v)=v-\left[A^{-1} \circ j_{t}^{*} \circ G^{\prime}(0)\right](v)=\operatorname{id}(v)=v$ (because $\left.G^{\prime}(0)=0\right)$ by $(2.11)$ we have

$$
\operatorname{deg}\left(\Phi, K\left(0, r_{0}\right), 0\right)=\operatorname{deg}\left(\Phi^{\prime}(0), K\left(0, r_{0}\right), 0\right)=1,
$$


where $r_{0}$ is small. Finally, by additivity (2.9),

$$
\sum_{i=1}^{s} \operatorname{deg}\left(\Phi, K\left(u_{i}, r_{i}\right), 0\right)=0
$$

provided $K\left(u_{i}, r_{i}\right) \subset K(0, R)$ for $i=1, \ldots, s$ and $K\left(u_{i}, r_{i}\right) \cap K\left(u_{j}, r_{j}\right)=\emptyset$ for $i \neq j$. Now the above equality and step $2^{\circ}$ finish the proof.

\section{References}

[1] H. Amann and S. A. Weiss, On the uniqueness of the topological degree, Math. Z. 130 (1973), 39-54.

[2] A. Ambrosetti and G. Mancini, Sharp nonuniqueness results for some nonlinear problems, Nonlinear Anal. 3 (1979), 635-645.

[3] A. A mbrosetti and P. H. Rabinowitz, Dual variational methods in critical point theory and applications, J. Funct. Anal. 14 (1973), 349-381.

[4] K.-C. Chang, Variational methods for non-differentiable functionals and their applications to partial differential equations, J. Math. Anal. Appl. 80 (1981), 102-129.

[5] A. Friedman, Partial Differential Equations, Holt, Rinehart and Winston, New York 1969.

[6] L. Gårding, Dirichlet's problem for linear elliptic partial differential equations, Math. Scand. 1 (1953), 55-72.

[7] E. M. Landesman and A. C. Lazer, Linear eigenvalues and a nonlinear boundary value problem, Pacific J. Math. 33 (1970), 311-328.

[8] A. C. Lazer and P. J. McKenna, On the number of solutions of a nonlinear Dirichlet problem, J. Math. Anal. Appl. 84 (1981), 282-294.

[9] L. Nirenberg, Topics in Nonlinear Functional Analysis, Courant Institute of Mathematical Sciences, New York University, 1974.

[10] R. S. Palais and S. Smale, A generalized Morse theory, Bull. Amer. Math. Soc. 70 (1964), 165-171.

[11] W. V. Petryshyn, Variational solvability of quasilinear elliptic boundary value problems at resonance, Nonlinear Anal. 5 (1981), 1095-1108.

[12] P. H. Rabinowitz, Minimax Methods in Critical Point Theory with Applications to Differential Equations, CBMS Regional Conf. Ser. in Math. 65, Amer. Math. Soc., Providence, R.I., 1986.

[13] M. Struve, A note on a result of Ambrosetti and Mancini, Ann. Mat. Pura Appl. 131 (1982), 107-115.

[14] M. Vaŭnberg, On the continuity of some operators of special type, Dokl. Akad Nauk SSSR 73 (1950), 253-255 (in Russian).

INSTITUTE OF MATHEMATICS

UNIVERSITY OF GDAŃSK

WITA STWOSZA 57

80-952 GDAŃSK, POLAND 